\title{
Vitamin A Deficiency Changes Jejunal Mucosal Fatty Acid Profile in Rats
}

\author{
Rosemary A. Warden, ${ }^{1, a}$ Rhiannon S. Noltore, ${ }^{2}$ C.J. Bell, ${ }^{3}$ E.V. O’LOUghlin, ${ }^{2, b}$ \\ P.R. DunKLEY, ${ }^{1}$ and Manohar L. GARG ${ }^{1, *}$ \\ ${ }^{1}$ Faculty of Medicine and Health Sciences, The University of Newcastle, Callaghan, NSW, \\ Australia \\ ${ }^{2}$ Department of Paediatrics, John Hunter Hospital, New Lambton Heights, NSW, Australia \\ ${ }^{3}$ Department of Gastroenterology, Royal North Shore Hospital, St Leonards, NSW, \\ Australia
}

(Received August 6, 2001)

Summary Changes in intestinal epithelial integrity have been postulated to explain the association between vitamin A status and diarrhoeal disease in children living in areas where vitamin A deficiency is a major public health problem. Fatty acids are an integral component of normal enterocyte structure, and changes to their profile in the intestinal mucosa may impact on the efficacy of the mucosal barrier. The aim of this study therefore was to determine the effect of vitamin A deficiency on intestinal mucosal fatty acid profile. Weanling male specific pathogen free rats were maintained on a vitamin A deficient diet for either 40-42 (subclinical deficiency) or 60-63 days (clinical deficiency), and compared with rats pair fed or allowed free access to the same diet, but supplemented with vitamin A (vitamin A sufficient). Fatty acid profile of jejunal mucosal homogenates was determined using gas chromatography. While subclinically vitamin A deficient rats had only minor differences in fatty acid profile as compared with pair fed and vitamin A sufficient rats, there were marked differences in a number of fatty acids in the clinically vitamin A deficient rats. Clinically vitamin A deficient rats had more arachidonic acid (C20:4n-6) than pair fed rats, and less linoleic acid (C18:2n-6) than both pair fed and vitamin A sufficient rats. These findings suggest that vitamin A deficiency modifies intestinal mucosal fatty acid composition such that there is an increase in proinflammatory eicosanoid precursors. This may contribute towards our understanding of a biological mechanism of the epidemiological association between vitamin A status and diarrhoeal disease.

* To whom correspondence should be addressed: Discipline of Nutrition \& Dietetics, Level 3, Medical Science Building, University of Newcastle, Callaghan, NSW 2308, Australia (Tel.: 61-24921-5647. Fax.: 61-2-4921-6984; E-mail: ndmg@mail.newcastle.edu.au).

Current address: ${ }^{a}$ Strategic Workforce Services, Territory Health Services, Casuarina, NT, Austra-

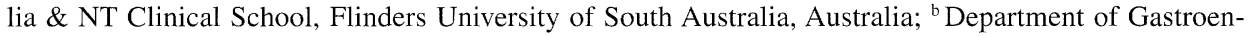
terology, The New Children's Hospital, Westmead, NSW, Australia. 
Key Words: vitamin A deficiency, rat, jejunum, fatty acids, eicosanoids

Improving the vitamin A status of children living in areas where vitamin A deficiency is a major public health problem, has been found to reduce both their overall mortality rate, and that specific to diarrhoeal disease [1-3]. In order to determine a physiological explanation for these findings, we have previously compared the effect of vitamin A deficiency on rat jejunal structure and function with pair-fed controls, and found that subclinical deficiency does not affect gross morphology, disaccharidase activities or glucose transport, whereas clinically deficient rats had mild villous atrophy and reduced disaccharidase activities (although this did not result in diarrhoea) [4]. However, in the presence of a noxious agent such as methotrexate [5] or rotavirus [6], vitamin A deficient rodents develop a more severe intestinal injury than controls treated with these agents. We have postulated that these findings may be due to impaired intestinal mucosal barrier function caused by vitamin A deficiency [5].

Indeed, altered intestinal epithelial integrity has been proposed as a possible mechanism for the epidemiological findings of an association between vitamin A status and diarrhoeal disease mortality in young children [1]. Epithelial integrity is an important factor in the maintenance of the intestinal mucosal barrier, which prevents the movement of pathogens, endotoxins, and antigens from the lumen into the systemic circulation and distant organs $[7,8]$.

Enterocyte cell structure (and function) is a component of intestinal epithelial integrity [8], and is influenced by membrane lipid composition $[9,10]$. The physicochemical and transport properties of both the brush border and basolateral membranes can vary with fatty acyl chain composition, cholesterol and phospholipid head group content [9, 11]. In turn, these components, and hence structural and metabolic activities of enterocytes, can be influenced by fasting and dietary manipulation $[9,10,12-15]$. While there has been interest in the possible interaction of vitamin A status and membranes for many years [16-19], and young rats given large doses of vitamin A have been found to have greater total intestinal lipid content and monoglycerides than controls [20,21], we are not aware of any studies conducted to investigate intestinal epithelial lipid composition in vitamin A deficiency.

The aims of these experiments were therefore to investigate the effect of both subclinical and clinical vitamin A deficiency on jejunal fatty acid composition.

\section{MATERIALS AND METHODS}

Animal models. An established rat model of subclinical and clinical vitamin A deficiency was used [4]. Specific pathogen free (SPF) weanling male Wistar rats (obtained direct or bred from the Animal Resources Centre, Perth, Australia), aged 3 weeks, were separated into three groups. Animals designated VA- were fed a pelleted vitamin A deficient diet (Catalog no: 960220, ICN Biomedicals, Sydney). Because consumption of this diet results in reduced food intake [4], the rats in the second group (PF) 
received the same diet, but were pair fed to those in the VA - group and supplemented with $150 \mathrm{mg}$ vitamin A (Rovimix A 500W, Roche Australia, Sydney) added to their drinking water (volume adjusted to thirst) every second day. To control for any effect of the vitamin A deficient diet, a third group ( $\mathrm{VA}+$ ) received the vitamin A deficient diet in unrestricted quantities, with their drinking water similarly supplemented with vitamin A. Rats were maintained on diets for either 40-42 days (subclinical deficiency) or 60-63 days (clinical deficiency) [4].

Rats were individually housed in wire bottom cages in a single room in a conventional animal house. The temperature was maintained at $20-22^{\circ} \mathrm{C}$, with a light-dark cycle of $12 \mathrm{~h}$. All animals had free access to water. Animals were observed for clinical evidence of vitamin A deficiency and the presence of diarrhoea. Rats were weighed on the day of study. All procedures were approved by the Animal Care and Ethics Committee, The University of Newcastle.

Mucosal fatty acid analysis. Fatty acid analyses were conducted on mucosal samples from a subset of a series of rats previously reported in studies of vitamin A deficiency and intestinal function and methotrexate enteropathy in rats $[4,5]$. On the day of study, animals were anaesthetized with intraperitoneal thiopentone $(0.16 \mathrm{~g} / \mathrm{kg})$ and blood collected by intracardiac puncture. Jejunum from the ligament of Trietz to mid jejunum was removed and flushed with cold phosphate-buffered saline. Mucosa was scraped off the distal half of this segment using two glass slides, homogenized in $2.5 \mathrm{mM}$ EDTA (1:10, weight:volume), snap frozen with liquid nitrogen and stored at $-80^{\circ} \mathrm{C}$ until analyzed.

For fatty acid determination, mucosal homogenate was thawed, and fatty acids were directly methylated in situ with acetyl chloride in toluene : methanol $(4: 1, \mathrm{v} / \mathrm{v})$, using a modification of the method of Lepage and Roy [22]. Fatty acid methyl esters were extracted and injected into a gas chromatograph (5890A, Hewlett Packard) equipped with a $30 \mathrm{~m}$ fused carbon-silica capillary column (internal diameter: $0.25 \mathrm{~mm}$ ) coated with cyanopropylphenyl (DB-225, 25 mm thickness, 25\% w/w coating; J \& W Scientific, CA), and connected to an integrator (3392A, Hewlett Packard). The initial temperature of $170^{\circ} \mathrm{C}$ was maintained for $2 \mathrm{~min}$, then increased to $210^{\circ} \mathrm{C}$ at a rate of $5^{\circ} \mathrm{C} / \mathrm{min}$, then to $212^{\circ} \mathrm{C}$ at a rate of $0.2^{\circ} \mathrm{C} / \mathrm{min}$, and finally to $220^{\circ} \mathrm{C}$ at $10^{\circ} \mathrm{C} / \mathrm{min}$. Fatty acid methyl ester peaks were identified by injecting standard mixtures of fatty acid methyl esters ( $\mathrm{Nu}$ Chek Prep Inc., Elysian, MN), and estimated using nonedecanoic acid (C19:0, Nu Chek Prep Inc.) as an internal standard. Contents of fatty acids are expressed as \%w/w. The Unsaturation Index (U.I.) was calculated as the sum of the number of double bonds in a given fatty acid multiplied by the amount (\%w/w) of that fatty acid present [12].

Retinol analyses. Serum retinol was measured by reversed-phase HPLC with UV detection as described previously [4].

Statistical analyses. Values are depicted as mean \pm SEM. For each of the subclinical and clinical studies, all groups were compared using one way analysis of variance or Kruskal-Wallis tests (for non parametric data). Where differences were detected, group means were compared using a Tukey-Kramer multiple comparison test or for non-parametric data, medians were compared using a Mann-Whitney test [23]. Differences between the subclinical and clinical studies were examined using two way analysis of 
variance, then either a Student $t$-test or Mann-Whitney test if differences were detected.

\section{RESULTS}

The clinical features of the groups of rats of which the animals in this study are a subset, have previously been described [4,5]. Rats consuming the vitamin A deficient diet for 60-63 days without vitamin A supplementation appeared dishevelled and emaciated. No rats from any other diet group showed similar signs of clinical vitamin A deficiency [4]. There was no significant difference in body weight between the subclinically VArats $(278 \pm 17.7 \mathrm{~g}), \mathrm{PF}(299.2 \pm 21.4 \mathrm{~g})$ and VA+ $(310 \pm 21.0 \mathrm{~g})$ controls. Clinically VArats weighed $(225.7 \pm 16.5 \mathrm{~g})$ significantly less than both $\mathrm{PF}(259.3 \pm 10.6 \mathrm{~g}, p<0.05)$ and $\mathrm{VA}+(313.9 \pm 11.0 \mathrm{~g}, p<0.001)$ controls.

\section{Serum retinol}

Serum retinol was negligible in subclinically VA - rats $(0.08 \pm 0.03 \mu \mathrm{mol} / \mathrm{liter})$, and was significantly less than PF $(2.19 \pm 0.13 \mu \mathrm{mol} / \mathrm{liter})$ and $\mathrm{VA}+(2.10 \pm 0.10 \mu \mathrm{mol} / \mathrm{liter})$ rats $(p<0.05)$. Clinically VA - rats had no detectable serum retinol, significantly less than both PF $(1.95 \pm 0.10 \mu \mathrm{mol} / \mathrm{liter})$ and $\mathrm{VA}+(1.77 \pm 0.24 \mu \mathrm{mol} / \mathrm{liter})$ rats $(p=0.02)$. There were no significant differences in serum retinol concentrations between the subclinical and clinical studies.

Table 1. Effects of subclinical vitamin A deficiency on fatty acid profile of jejunal mucosa.

\begin{tabular}{lccc}
\hline $\begin{array}{c}\text { Fatty acid } \\
\text { \%w/w }\end{array}$ & $\begin{array}{c}\text { Vitamin A deficient } \\
(\text { VA }-)\end{array}$ & $\begin{array}{c}\text { Pair fed } \\
(\mathrm{PF})\end{array}$ & $\begin{array}{c}\text { Vitamin A sufficient } \\
(\text { VA }+)\end{array}$ \\
\hline C14:0 & $n=9$ & $n=9$ & $n=10$ \\
C15:0 & $0.8 \pm 0.1$ & $0.8 \pm 0.1$ & $0.8 \pm 0.1$ \\
C16:0 & $0.6 \pm 0.1^{\mathrm{a}}$ & $0.4 \pm 0.1^{\mathrm{b}}$ & $0.5 \pm 0.0$ \\
C16:1n-7 & $23.5 \pm 0.6^{\mathrm{a}}$ & $21.5 \pm 0.5^{\mathrm{b}}$ & $21.3 \pm 0.3^{\mathrm{b}}$ \\
C17:0 & $2.2 \pm 0.5$ & $2.1 \pm 0.4$ & $2.2 \pm 0.3$ \\
C18:0 & $0.8 \pm 0.1$ & $0.7 \pm 0.1$ & $0.7 \pm 0.1$ \\
C18:1n-9 & $13.5 \pm 1.2$ & $13.9 \pm 1.3$ & $14.9 \pm 0.9$ \\
C18:1n-7 & $13.7 \pm 1.4$ & $14.5 \pm 1.6$ & $13.6 \pm 1.2$ \\
C18:2n-6 & $2.5 \pm 0.2$ & $2.4 \pm 0.1$ & $2.6 \pm 0.1$ \\
C20:0 & $23.6 \pm 0.8$ & $26.4 \pm 2.0$ & $25.4 \pm 1.0$ \\
C20:1n-9 & $0.5 \pm 0.0$ & $0.5 \pm 0.0$ & $0.5 \pm 0.0$ \\
C20:2n-6 & $0.2 \pm 0.0$ & $0.2 \pm 0.0$ & $0.2 \pm 0.0$ \\
C20:3n-6 & $0.3 \pm 0.0$ & $0.4 \pm 0.0$ & $0.4 \pm 0.0$ \\
C20:4n-6 & $0.6 \pm 0.1$ & $0.8 \pm 0.1$ & $0.8 \pm 0.1$ \\
C22:0 & $14.4 \pm 1.2$ & $13.0 \pm 2.1$ & $13.6 \pm 1.4$ \\
C22:4n-6 & $0.3 \pm 0.1$ & $0.4 \pm 0.1$ & $0.5 \pm 0.1$ \\
C22:6n-3 & $0.9 \pm 0.1$ & $1.1 \pm 0.1$ & $1.1 \pm 0.1$ \\
C24:0 & $0.4 \pm 0.1$ & $0.3 \pm 0.1$ & $0.3 \pm 0.0$ \\
\hline Unsaturation index & $132.6 \pm 3.1$ & $0.4 \pm 0.0$ & $0.4 \pm 0.0$ \\
\hline
\end{tabular}

Values are mean \pm SEM. Values in a column with a different superscript letter are significantly different $(p<0.05)$. 
Jejunal mucosal fatty acid analysis

In the subclinical study, no major differences were detected in jejunal mucosal fatty acid profile (Table 1). However VA - mucosal homogenate did have significantly higher percentages of C15:0 and C16:0 fatty acids than PF $(p<0.05$, Table 1$)$, and significantly higher percentages of $\mathrm{C} 16: 0$ fatty acids than VA+ $(p<0.05$, Table 1$)$. There were no differences in U.I. between groups (Table 1).

In clinical vitamin A deficiency, major differences were detected between groups in fatty acid profile: VA - mucosal homogenate had significantly less C18:2n-6 than PF and $\mathrm{VA}+(p<0.02$, Table 2$)$ and significantly more C20:4n-6 than PF $(p<0.02$, Table 2$)$. VA - mucosal homogenate also had significantly more C15:0, C16:0, C18:1n-7, C20:0, C20:1n-9, C22:4n-6, C22:6n-3 and C24:0 than PF ( $p<0.02$, Table 2). Mucosal homogenate from VA - group rats also had significantly more C14:0, C16:0, C20:0, C20:1n-9, C22:0, C22:4n-6, C22:6n-3 and C24:0; and significantly less C18:2n-6 and C20:2n-6 than $\mathrm{VA}+(p<0.02$, Table 2$)$. PF animals had significantly less $\mathrm{C} 18: 1 \mathrm{n}-7$ than VA+ $(p<0.02$, Table 2$)$. There were no differences in U.I. between groups (Table 2).

\section{DISCUSSION}

Clinical vitamin A deficiency caused major alterations in jejunal mucosal fatty acid profile. Specifically, there was more arachidonic acid (C20:4n-6) and less linoleic acid

Table 2. Effects of clinical vitamin A deficiency on fatty acid profile of jejunal mucosa.

\begin{tabular}{lccc}
\hline \multicolumn{1}{c}{ Fatty acid } & $\begin{array}{c}\text { Vitamin A deficient } \\
\text { (VA } / \mathrm{w}\end{array}$ & $\begin{array}{c}\text { Pair fed } \\
(\text { PF })\end{array}$ & $\begin{array}{c}\text { Vitamin A sufficient } \\
(\text { VA }+)\end{array}$ \\
\cline { 2 - 4 } C14:0 & $n=8$ & $n=8$ & $n=8$ \\
C15:0 & $0.9 \pm 0.1^{\mathrm{a}}$ & $0.7 \pm 0.1^{\mathrm{ab}}$ & $0.7 \pm 0.0^{\mathrm{b}}$ \\
C16:0 & $0.7 \pm 0.1^{\mathrm{a}}$ & $0.4 \pm 0.0^{\mathrm{b}}$ & $0.6 \pm 0.1^{\mathrm{ab}}$ \\
C16:1n-7 & $23.6 \pm 0.6^{\mathrm{a}}$ & $21.3 \pm 0.6^{\mathrm{b}}$ & $19.8 \pm 0.4^{\mathrm{b}}$ \\
C17:0 & $2.2 \pm 0.2$ & $1.5 \pm 0.2$ & $1.7 \pm 0.2$ \\
C18:0 & $0.8 \pm 0.1$ & $0.6 \pm 0.1$ & $0.8 \pm 0.1$ \\
C18:1n-9 & $13.4 \pm 0.7$ & $10.4 \pm 1.7$ & $13.4 \pm 0.4$ \\
C18:1n-7 & $13.8 \pm 1.3$ & $14.3 \pm 0.8$ & $12.8 \pm 0.6$ \\
C18:2n-6 & $2.8 \pm 0.1^{\mathrm{a}}$ & $2.1 \pm 0.2^{\mathrm{b}}$ & $2.6 \pm 0.1^{\mathrm{a}}$ \\
C20:0 & $18.7 \pm 0.9^{\mathrm{a}}$ & $33.8 \pm 1.9^{\mathrm{b}}$ & $29.2 \pm 0.9^{\mathrm{b}}$ \\
C20:1n-9 & $0.6 \pm 0.0^{\mathrm{a}}$ & $0.5 \pm 0.0^{\mathrm{b}}$ & $0.5 \pm 0.0^{\mathrm{b}}$ \\
C20:2n-6 & $0.3 \pm 0.0^{\mathrm{a}}$ & $0.2 \pm 0.0^{\mathrm{b}}$ & $0.2 \pm 0.0^{\mathrm{b}}$ \\
C20:3n-6 & $0.4 \pm 0.0^{\mathrm{a}}$ & $0.4 \pm 0.0^{\mathrm{a}}$ & $0.5 \pm 0.0^{\mathrm{b}}$ \\
C20:4n-6 & $0.7 \pm 0.1$ & $0.6 \pm 0.0$ & $0.6 \pm 0.0$ \\
C22:0 & $17.2 \pm 1.6^{\mathrm{a}}$ & $10.8 \pm 0.9^{\mathrm{b}}$ & $14.0 \pm 0.6^{\mathrm{ab}}$ \\
C22:4n-6 & $0.4 \pm 0.0^{\mathrm{a}}$ & $0.4 \pm 0.0^{\mathrm{a}}$ & $0.3 \pm 0.0^{\mathrm{b}}$ \\
C22:6n-3 & $1.7 \pm 0.1^{\mathrm{a}}$ & $1.0 \pm 0.1^{\mathrm{b}}$ & $1.2 \pm 0.1^{\mathrm{b}}$ \\
C24:0 & $0.5 \pm 0.1^{\mathrm{a}}$ & $0.2 \pm 0.0^{\mathrm{b}}$ & $0.3 \pm 0.0^{\mathrm{b}}$ \\
\hline Unsaturation index & $0.5 \pm 0.0^{\mathrm{a}}$ & $0.4 \pm 0.0^{\mathrm{b}}$ & $0.3 \pm 0.0^{\mathrm{b}}$ \\
\hline Values are mean \pm SEM. Values in a column with a different superscript letter are significantly dif- & & \\
ferent $(p<0.02)$. & $139.1 \pm 4.5$ & & $141.8 \pm 0.9$
\end{tabular}


(C18:2n-6) than in pair fed controls. In contrast, a tendency of more arachidonic acid and less linoleic acid was detected in subclinical vitamin A deficient rats.

Interestingly, we can also hypothesize that there may be a functional link between our findings of altered intestinal mucosal fatty acid profile and disaccharidase activities in the clinically deficient rats. Changes in fatty acid composition of microsomal membrane phospholipids may influence alterations to membrane cholesterol and phospholipid biosynthesis, which are important membrane (and hence enterocyte) structural and functional determinants [11]. Although we have previously postulated that changes observed in disaccharidase activities in clinically vitamin A deficient rats may be attributed to vitamin A's role in gene expression [4], changes in membrane-order parameters have also been noted to alter membrane-bound hydrolytic enzyme activity [11], and therefore should be considered as a possible mechanism for our earlier findings.

Although clinically vitamin A deficient rats were more malnourished than subclinically deficient rats [4], it is unlikely that our findings at each stage of deficiency are due to any excess effect of malnutrition, as we have controlled for the effect of reduced intake due to consumption of a vitamin A deficient diet [4] by pair-feeding one group of controls. Although no studies have examined the effect of protein energy malnutrition on intestinal fatty acid profile, malnourished rabbits have an altered intestinal microvillous membrane lipid composition [13], and short-term fasting in rats has been found to modify the fatty acid profile of intestinal microsomes [12]. Furthermore, by feeding the same diet to all groups of rats, we have also controlled for the effects of any other nutrients which may influence intestinal mucosal fatty acid composition $[9,10,12,14,15]$.

The differences in fatty acid profile in clinically vitamin A deficient rats may have a major impact on the nature of eicosanoids synthesized in the intestinal mucosa. Clinically vitamin A deficient rats have significantly more arachidonic acid (C20:4n-6) than PF rats. Arachidonic acid is a precursor of eicosanoids such as prostaglandins (eg. $\mathrm{PGE}_{2}$ ), thromboxane, and leukotrienes; all implicated in the pathogenesis of intestinal inflammation [24]. Although the few studies addressing the addition of retinoids to mesenchymal or endothelial cell cultures have found either no difference [25], or a decrease [26] in $\mathrm{PGE}_{2}$ production, no studies have investigated the effect of vitamin A deficiency on eicosanoid production.

Clinically vitamin A deficient rats also have less linoleic acid (C18:2n-6) than both pair fed and vitamin A sufficient rats. Linoleic acid is converted to linolenic acid by $\Delta^{6}$ desaturase, and the same enzyme is also the rate limiting step in arachidonic acid synthesis from linoleic acid [9]. Thus it is likely that intestinal $\Delta^{6}$ desaturase is upregulated during vitamin A deficiency. $\Delta^{6}$ Desaturase has been found in intestinal epithelia [12], and its activity in rat small intestinal mucosal microsomes has been shown to be altered by both short term fasting and dietary fat manipulation [12]. Vitamin A deficient rats have been found to have increased liver microsomal $\Delta^{9}$, but not $\Delta^{6}$, desaturase activity [27], and reduced arachidonic, but increased palmitic acid content as compared with controls [28]. However, $\Delta^{6}$ desaturase activity appears to be regulated differently in the liver than in the intestine [11], and additionally, Alam and Alam [28] did not use pair fed controls in their studies. 
Others [19, 29] have also reported changes in liver microsomal fatty acid profile in vitamin A deficient rats, although the changes differed between studies. Furthermore, both of these studies also examined microsomal membrane fluidity, and found that it was decreased in vitamin A deficiency. Grolier et al. [19] postulate that this may be due to observed changes in phospholipid to cholesterol ratio and phospholipid content. Given that high doses of vitamin A have been found to alter total intestinal lipid content [20, 21], vitamin A deficiency may also cause changes in intestinal epithelial membrane fluidity, potentially resulting in changes in the membrane's barrier function.

A tendency to increase production of pro-inflammatory eicosanoids, predisposing the mucosa to inflammatory injury, may explain our previous findings and those of others that vitamin A deficiency exacerbates methotrexate enteropathy [5] and rotavirus enteritis [6]. Moreover, in children whose intestines are regularly insulted with pathogens and other antigens, an overproduction of pro-inflammatory eicosanoids causing increased susceptibility to intestinal mucosal damage, is a possible biological explanation for the epidemiological association of vitamin A deficiency and diarrhoeal disease.

The authors gratefully acknowledge Bill Ebeling for technical assistance with the vitamin A analyses, Robert Blake for technical assistance with the fatty acid analyses, and Sue Maastricht and Anne Feighan for their assistance with the rat experiments. Supported in part by grants from the Research Management Committee of the University of Newcastle, and the John Hunter Hospital Staff Specialists Charitable Trust. Rovimix A 500W was a gift from Roche, Australia.

\section{REFERENCES}

1. Beaten, G.H., Martorell, R., and Aronson, K.J. (1993): Effectiveness of vitamin A supplementation in the control of young child morbidity and mortality in developing countries. ACC/SCN State of the art series nutrition policy discussion paper no.13. The Lavenham Press Ltd., Suffolk, UK.

2. Fawzi, W.W., Chalmers, T.C., Herrera, G.M., and Mosteller, F. (1993): Vitamin A supplementation and child mortality. A meta-analysis. J. Am. Med. Assoc., 269, 898-903.

3. Glasziou, P.P., and Mackerras, D.E.M. (1993): Vitamin A supplementation in infectious diseases: Meta-analysis. Br. Med. J., 306, 366-370.

4. Warden, R.A., Strazzari, M.J., Dunkley, P.R., and O'Loughlin, E.V. (1996): Vitamin A-deficient rats have only mild changes in jejunal structure and function. J. Nutr. 126, 1817-1826.

5. Warden, R.A., Noltorp, R.S., Francis, J.L., Dunkley, P.R., and O'Loughlin, E.V. (1997): Vitamin A deficiency exacerbates methotrexate-induced jejunal injury in rats. J. Nutr., 127, 770-776.

6. Ahmed, F., Jones, D.B., and Jackson, A.A. (1990): The interaction of vitamin A deficiency and rotavirus infection in the mouse. Br. J. Nutr., 63, 363-373.

7. Deitch, E.A. (1994): Bacterial translocation: The influence of dietary variables. Gut, Suppl. 1., S23S27.

8. Van Leeuwen, P.A., Boermeester, M.A., Houdijk, A.P., Ferwerda, C.C., Cuesta, M.A., Meyer, S., and Wesdrop, R.I. (1994): Clinical significance of translocation. Gut, 35, S28-34.

9. Clandinin, M.T., Cheema, S., Field, C.J., Garg, M.L., Venkatraman, J., and Clandinin, T.R. (1991): Dietary fat: Exogenous determination of membrane structure and cell function. FASEB J., 5, 27612769.

10. Meddings, J.B., and Theisen, S. (1989): Development of rat jejunum: Lipid permeability, physical properties, and chemical composition. Am. J. Physiol., 256, G931-G940.

11. Garg, M.L., Keelan, M., Thomson, A.B.R., and Clandinin, M.T. (1990): Intestinal microsomes: Polyunsaturated fatty acid metabolism and regulation of enterocyte transport properties. Can. J. Physiol. Pharmacol., 68, 636-641. 
12. Garg, M.L., Keelan, M., Thomson, A.B.R., and Clandinin, M.T. (1992): Desaturation of linoleic acid in the small bowel is increased by short-term fasting and by dietary content of linoleic acid. Biochim. Biophys. Acta, 1126, 17-25.

13. Butzner, J.D., Brockway, P.D., and Meddings, J.B. (1990): Effects of malnutrition on microvillus membrane glucose transport and physical properties. Am. J. Physiol., 259, G940-G946.

14. Christon, R., Meslin, J.C., Thevenoux, J., Linard, A., Leger, C.L., and Delpal, S. (1991): Effects of a low dietary linoleic acid level on intestinal morphology and enterocyte brush border membrane lipid composition. Reprod. Nutr. Dev., 31, 691-701.

15. Clejan, S., Castro-Magana, M., Collipp, P.J., Jonas, E., and Maddaiah, V.T. (1982): Effects of zinc deficiency and castration on fatty acid composition and desaturation in rats. Lipids, 17, 129-135.

16. Roels, O.A., Anderson, O.R., Lui, N.S.T., Shah, D.O., and Trout, M.E. (1969): Vitamin A and membranes. Am. J. Clin. Nutr., 22, 1020-1032.

17. Lucy, J.A. (1969): Some possible roles for vitamin A in membranes: Micelle formation and electron transfer. Am. J. Clin. Nutr., 22, 1033-1044.

18. Stillwell, W., Ricketts, M., Hudson, H., and Nahmias, S. (1982): Effect of retinol and retinoic acid on permeability, electrical resistance and phase transition of lipid bilayers. Biochim. Biophys. Acta, $\mathbf{6 8 8}$, 653-659.

19. Grolier, P., Cisti, A., Daubeze, M., and Narbonne, J.F. (1991): The influence of dietary vitamin A intake on microsomal membrane fluidity and lipid composition. Nutr. Res., 11, 567-574.

20. Misra, U.K. (1967): Effect of hypervitaminosis A on intestinal lipids of rats. Can. J. Biochem., 45, 1413-1418.

21. Misra, U.K. (1966): Lipid metabolism in hypervitaminosis A. Nature, 209, 910-911.

22. Lepage, G., and Roy, C.C. (1986): Direct transesterification of all classes of lipids in a one-step reaction. J. Lipid Res., 27, 114-120.

23. Sokal, R.R., and Rohlf, F.J. (1981): Biometry, 2nd ed., W.H. Freeman, San Francisco, CA.

24. O'Loughlin, E.V., and Gall, D.G. (1996): Pathophysiology of intestinal inflammation, in Pediatric Gastrointestinal Disease: Pathophysiology, Diagnosis, Management, ed. by Walker, W.A., Durie, P.R., Hamilton, J.R., Walker-Smith, J.A., and Watkins, J.B., 2nd ed., Mosby, St. Louis, pp. 624-644.

25. Spencer-Green, G. (1994): Retinoic acid effects on endothelial cell function: Interaction with interleukin 1. Clin. Immun. Immunopath., 72, 53-61.

26. Brinckerhoff, C.E., McMillan, R.M., Dayer, J.-M., and Harris, E.D., Jr. (1980): Inhibition by retinoic acid of collegenase production in rheumatoid synovial cells. N. Eng. J. Med., 303, 432-436.

27. Alam, S.Q., and Alam, B.S. (1985): Microsomal fatty acid desaturase activities in vitamin A-deficient rat liver. Biochim. Biophys. Acta, 833, 175-177.

28. Gorgoshidze, L.S., Kon'IIa, Kulakova, S.N., and Sheviakov, A.N. (1986): Lipid peroxidation in the rat liver in mild forms of vitamin A deficiency. Vopr. Pitan., Sept-Oct, 45-50.

29. Hamm, M.W., Chan, V., and Wolf, G. (1987): Liver microsomal fluidity and lipid characteristics in vitamin A-deficient rats. Biochem. J., 245, 907-910. 